\title{
An unusual case of nonbacterial thrombotic (marantic) endocarditis
}

Cevher Ozcan, MD, Edward J. Miller, MD, Kerry S. Russell, MD, PhD, Michael L. Dewar, MD, and Lynda E. Rosenfeld, MD, New Haven, Conn

Nonbacterial thrombotic (marantic) endocarditis (NBTE) is a rare clinical condition manifest as various-sized cardiac valvular lesions ranging from microscopic aggregates of platelets to large vegetations of fibrin and platelets. It is difficult to diagnose NBTE before death. Despite significant advances in noninvasive diagnostic techniques, definitive diagnosis requires a tissue biopsy. We present an unusual case of NBTE with a large subvalvular mass invading the left ventricular posterior wall and papillary muscles and causing mitral stenosis. In this case, the final diagnosis was only made at the time of therapeutic surgical excision of the mass and mitral valve replacement.

\section{CLINICAL SUMMARY}

A 30-year-old woman with a history of aplastic anemia and an HLA-matched allogenic stem cell transplantation 2 years before admission had fever and hypoxemia. On physical examination she had diffuse rales and a diastolic murmur. Her course had been complicated by chronic graft-versus-host disease (GVHD) with fasciitis, treated with prednisone and sirolimus. Computed tomography of the chest revealed pulmonary edema with bilateral pleural effusions and superimposed pneumonia. Blood cultures were sterile. Tests for the human immunodeficiency virus, cytomegalovirus, Epstein-Barr virus, Q fever, Chlamydia, Mycoplasma, Bartonella, and Brucella species were negative. Antinuclear antibody and anti-double-stranded DNA tests were positive, whereas rheumatoid factor and anticardiolipin antibody tests were negative. Echocardiography showed a layered mass occupying the space between the posterior mitral leaflet and the left ventricular wall. It extended from the anterior to the posterior papillary muscle (Figure $1, A$ and $B$ ), restricting the opening of the mitral valve and producing a mean forward flow gradient of 9.8 $\mathrm{mm} \mathrm{Hg}$ (Figure 1, $C$ and $D$ ). Cardiac magnetic resonance imaging revealed that this region was iso-intense to myocardium and consistent with an infiltrative process. The patient became afebrile after empiric antibiotic treatment. An initial intense eosinophilia resolved after an increase in the long-term prednisone therapy. The possibility of a cardiac

\footnotetext{
From the Section of Cardiovascular Medicine, Yale University School of Medicine, New Haven, Conn

Received for publication Dec 13, 2007; revisions received Jan 3, 2008; accepted for publication Jan 20, 2008.

Address for reprints: Lynda E. Rosenfeld, MD, Section of Cardiovascular Medicine 3FMP, Yale University School of Medicine, 333 Cedar St, New Haven, CT 06510 (E-mail: lynda.rosenfeld@yale.edu).

J Thorac Cardiovasc Surg 2009;137:239-41

$0022-5223 / \$ 36.00$

Copyright (c) 2009 by The American Association for Thoracic Surgery

doi:10.1016/j.jtcvs.2008.01.019
}

tumor or lymphoma was considered, and positron emission tomography with flurodeoxyglucose was performed. There was no evidence of enhanced flurodeoxyglucose uptake in the mass or elsewhere in the body. Because of the hemodynamic significance of the mass and the possibility of tumor or thrombus, the patient underwent heart surgery. The mass was excised and the mitral valve replaced. Gross and microscopic histopathologic examination demonstrated organized fibrin-platelet thrombus adherent to the mitral valve and encasing the chordae tendineae (Figure 2, $A$ to $D)$. No organisms, fungi, or tumor cells were identified. There were foci of chronic inflammation with plasma cells, mast cells, lymphocytes, and eosinophils. Culture-negative endocarditis was excluded histologically on the basis of the lack of acute inflammation and the absence of fungi or bacteria. Thus these findings were consistent with NBTE, most likely resulting from the bone morrow transplantation and/ or chronic GVHD. The patient's postoperative course was uncomplicated.

\section{DISCUSSION}

NBTE, first described in the late 19th century, is the result of spontaneous fibrin-platelet deposition within the heart. ${ }^{1}$ The true incidence of this condition is difficult to assess inasmuch as the diagnosis is rarely made during life. Autopsy series have shown that the incidence of NBTE varies widely, from $0.3 \%$ to $9.3 \%$. $^{1}$ Endocardial injury and a hypercoagulable state are the two main mechanisms postulated for platelet-thrombin deposition, usually on the atrial surface of the mitral and tricuspid valves or the ventricular surface of the aortic or pulmonic valves. The altered valve tissue swells and ulcerates, and vegetations develop on the sites of rupture of the endothelial surface with extension into the involved valve leaflet. ${ }^{1,2}$ It is unusual for NBTE to invade the ventricular surface of the mitral valve or to cause a large left ventricular mass. NBTE has been associated with malignancy, other wasting disorders, disseminated intravascular coagulopathy, and autoimmune disorders. ${ }^{1-3}$ The prevalence of NBTE in bone marrow transplant recipients is increased $(7.7 \%)$ but not associated with an excess of GVHD. ${ }^{4}$ However, GVHD, which is associated with a hypercoagulable state and chronic inflammation, may contribute to NBTE. The definitive diagnosis proved particularly challenging in this patient. Infective endocarditis with unusual pathogens and a secondary malignant tumor were considered. Early diagnosis and treatment may reduce the morbidity and mortality of NBTE. NBTE may be associated with significant complications, particularly arterial or pulmonary embolization. Noninvasive imaging provided important but 

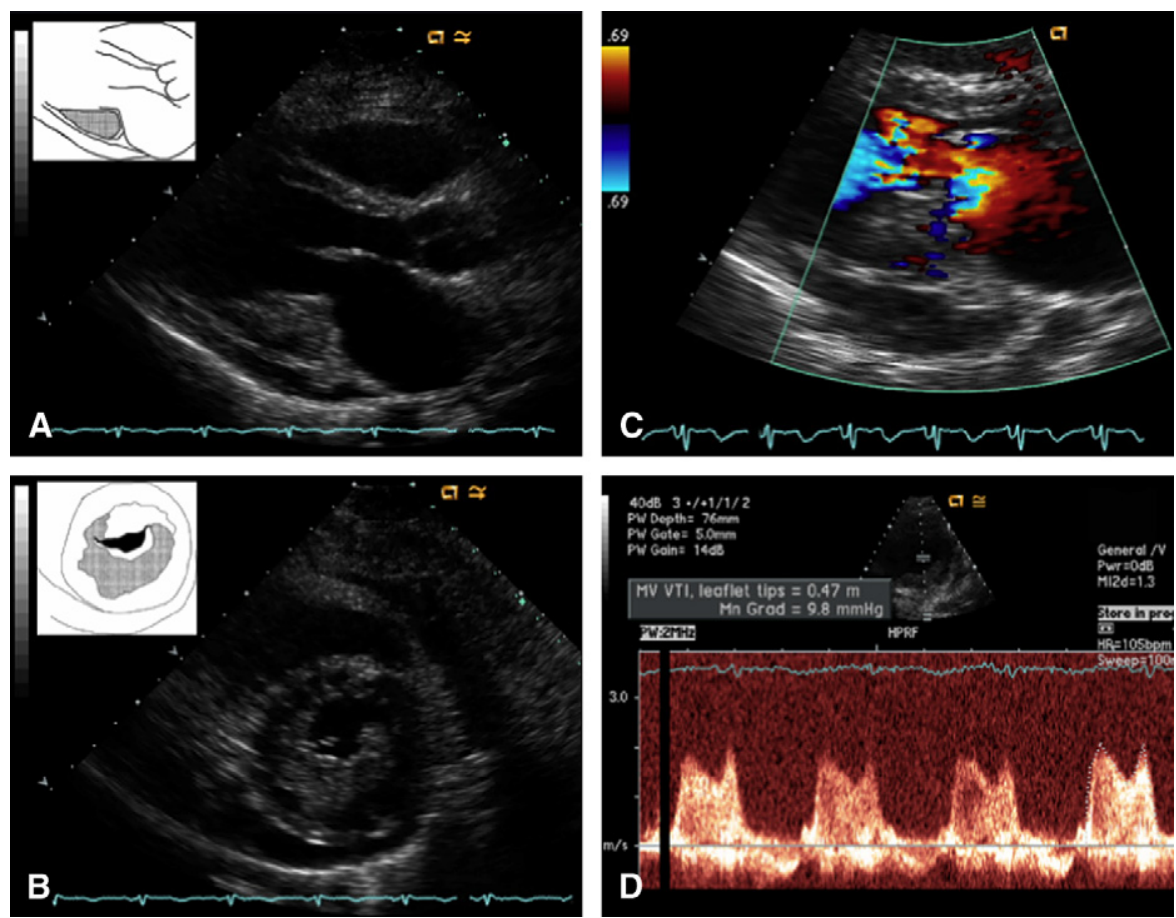

FIGURE 1. Transthoracic echocardiogram. Parasternal long-axis (A) and short-axis (B) views demonstrating a subvalvular mass between the posterior mitral valve leaflet and the posterior left ventricular wall, as well as restricted opening of the posterior leaflet of the mitral valve. Diagrams (insets) highlight location of the mass (hatched area). A small circumferential pericardial effusion was present. C, Color flow imaging of mitral valve showing mild mitral regurgitation with moderate mitral stenosis. D, Continuous Doppler recording demonstrating a peak mitral velocity $(M V V T I)$ of $2.0 \mathrm{~m} / \mathrm{s}$ and a mean transmitral forward flow gradient (Mn Grad) of $9.8 \mathrm{~mm} \mathrm{Hg}$.
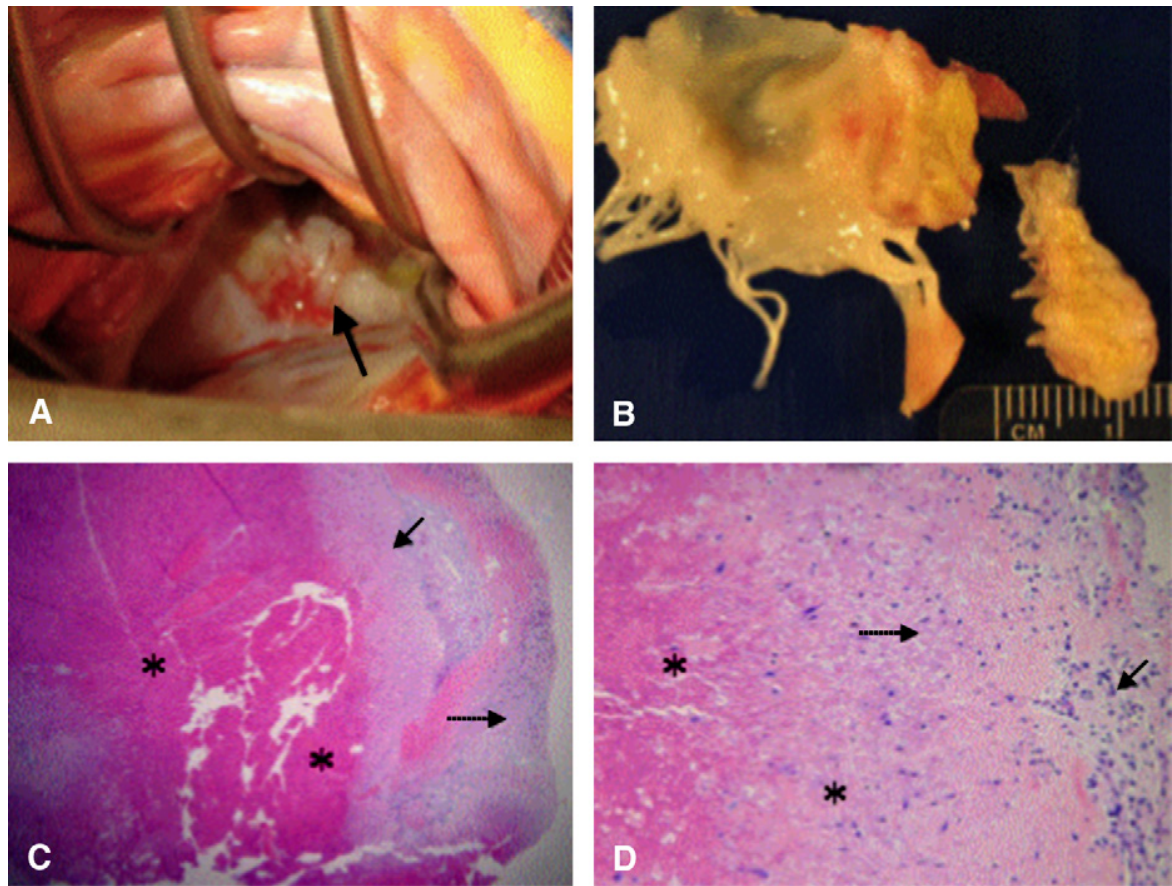

FIGURE 2. A, Surgical view of mitral valve and mass (arrow). B, Gross surgical pathologic specimen of the explanted mitral valve with mass. C and D, Histologic sections (hematoxylin and eosin, low and medium power) of the mass biopsy specimen showing organized fibrin-platelet thrombus (asterisks) foci of chronic inflammation with lymphocytes, plasma cells, mast cells and eosinophils (arrows). 
inconclusive information about the etiology of the mass in our case, and catheter-based biopsy was not considered possible. Therefore, the patient underwent cardiac surgery for definitive diagnosis and treatment. Our case is unusual in that the NTBE involved the ventricular surface of the mitral valve, invaded the left ventricular wall, resulted in a large mass, caused significant mitral stenosis, and was diagnosed before death.

\section{References}

1. Lopez AJ, Ross RS, Fishbein MC, Siegel RJ. Nonbacterial thrombotic endocarditis: a review. Am Heart J. 1987;113:773-84.

2. Waller BF, Knapp WS, Edwards JE. Marantic valvular vegetations. Circulation 1973;48:644-50.

3. Eiken PW, Edwards WD, Tezelaar HD, McBane RD, Zehr KJ. Surgical pathology of nonbacterial thrombotic endocarditis in 30 patients, 1985-2000. Mayo Clin Proc. 2001;76:1204-12.

4. Patchell RA, White 3rd CL, Clark AW, Beschorner WE, Santos GW. Nonbacterial thrombotic endocarditis in bone morrow transplant patients. Cancer. 1985;55:631-5. 